Supporting Information 


\section{Ionic Carbazole-Based Water-Soluble Two- Photon Photoinitiator and the Fabrication of Biocompatible 3D Hydrogel Scaffold}

Wen Gao ${ }^{1,3}$, Hao Chao ${ }^{2}$, Yong-Chao Zheng ${ }^{1}$, Wei-Cai Zhang ${ }^{1,3}$, Jie Liu ${ }^{1}$, Feng Jin, ${ }^{1}$ Xian-Zi Dong, ${ }^{1}$ Yan-Hong Liu ${ }^{1}$, Shu-Jing Li $^{2}$, Mei-Ling Zheng ${ }^{1,3, *}$

${ }^{1}$ Laboratory of Organic NanoPhotonics and CAS Key Laboratory of Bio-Inspired Materials and Interfacial Science, Technical Institute of Physics and Chemistry, Chinese Academy of Sciences, No. 29 Zhongguancun East Road, Beijing, 100190, P. R. China ${ }^{2}$ Department of Chemistry, School of Materials Science and Chemical Engineering, Beijing Technology and Business University, No. 11 Fucheng Road, Beijing, 100048, P. R. China

${ }^{3}$ School of Future Technologies, University of Chinese Academy of Sciences, Yanqihu Campus, Huaibei Zhuang, Huaibei Town, Huairou District, Beijing, 101407, P. R.

China

Corresponding Author

*Emial: zhengmeiling@mail.ipc.ac.cn 


\section{Contents}

1. ${ }^{1} \mathrm{H}$ NMR of BMVMC

2. Home-built femtosecond laser two-photon polymerization direct writing system

3. Computational methods

4. Fluorescence titration of BMVMC and CB7

5. The quadratic dependence during TPA cross section measurement

6. The electron paramagnetic resonance (EPR) characterization of CB7/BMVMC aqueous solution

7. Cell viability of CB7/BMVMC aqueous solution 


\section{1. ${ }^{1} \mathrm{H}$ NMR of BMVMC}

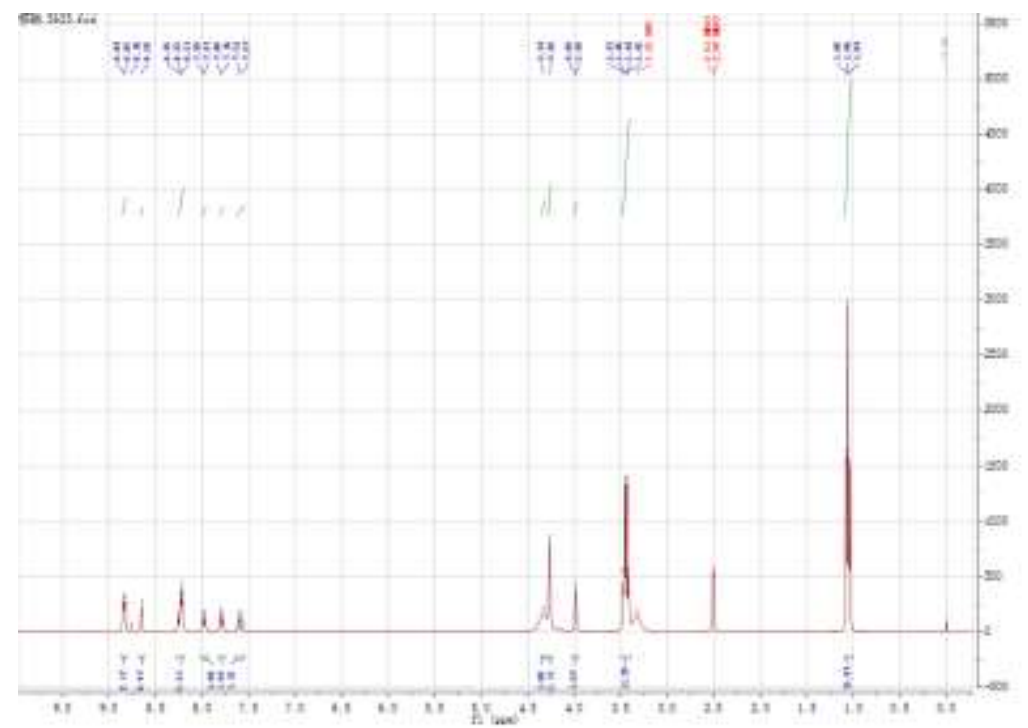

Figure S1. ${ }^{1} \mathrm{H}$ NMR of BMVMC (400 MHz,DMSO-d6, ppm) 3.99(d, 3H, J=4 Hz), 4.26(S, 2H), $7.59(\mathrm{~d}, 2 \mathrm{H}, \mathrm{J}=16 \mathrm{~Hz}), 7.79(\mathrm{~d}, 2 \mathrm{H}, \mathrm{J}=8 \mathrm{~Hz}), 7.98(\mathrm{~d}, 2 \mathrm{H}, \mathrm{J}=8 \mathrm{~Hz}), 8.23(\mathrm{~m}, 5 \mathrm{H}), 8.65(\mathrm{~s}, 2 \mathrm{H}), 8.84(\mathrm{~d}$, $4 \mathrm{H}, \mathrm{J}=4 \mathrm{~Hz})$. 
2. Home-built femtosecond laser two-photon polymerization direct writing system

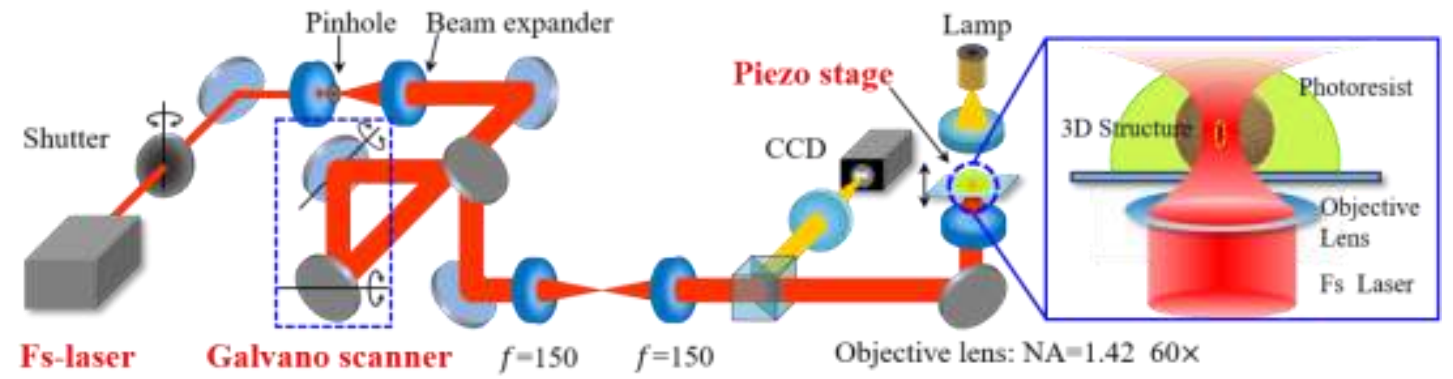

Figure S2. Schematic of the laser direct writing system. 
3. Computational methods

(a)

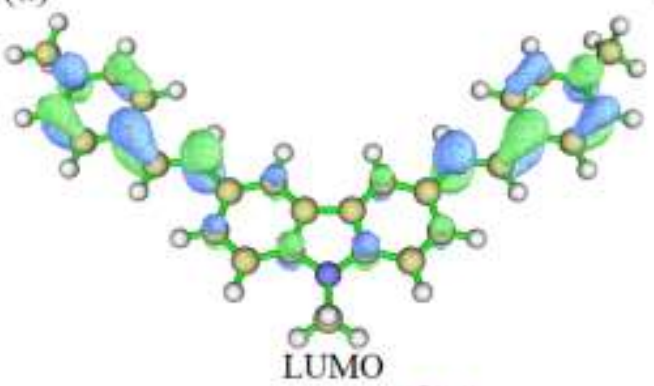

(c)

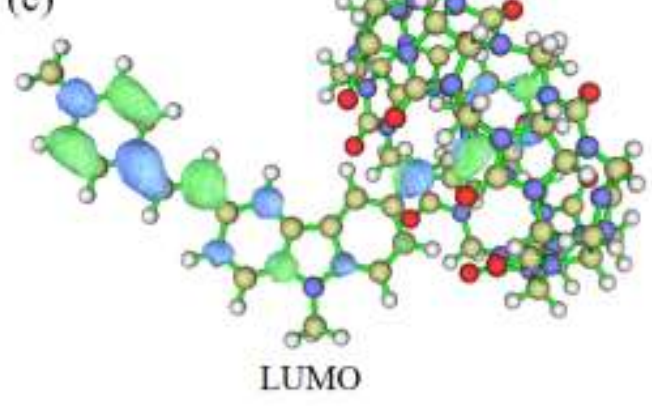

(b)

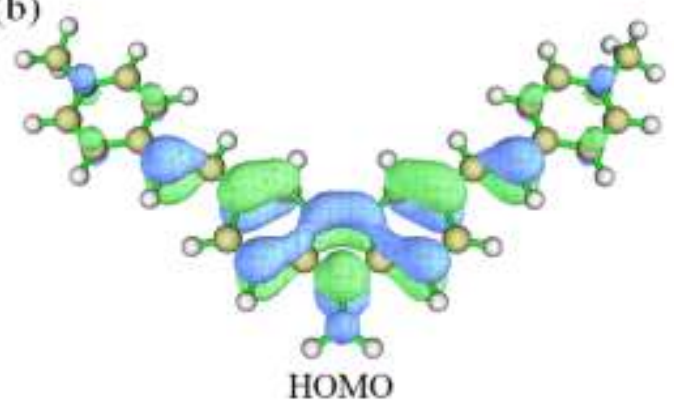

(d)

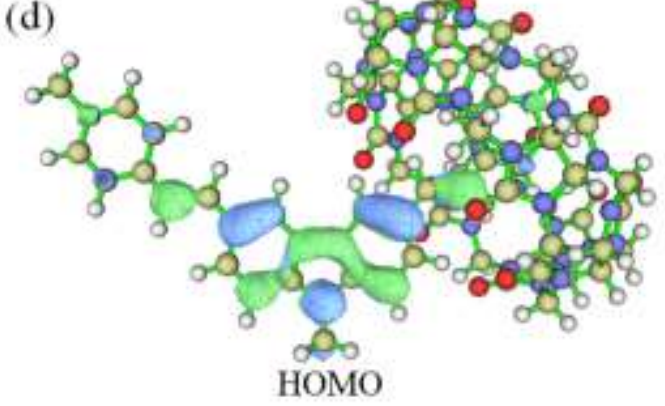

Figure S3 (a) LUMO and (b) HOMO orbitals of BMVMC, (c) LUMO and (d) HOMO orbitals of CB7/BMVMC with optimized Geometry. 


\section{Fluorescence titration of BMVMC and CB7}

Fluorescence spectra were measured by a Hitachi F-4600 spectrometer at room temperature $(25 \square)$, with the excitation wavelength of $450 \mathrm{~nm}$. Quartz cells of $1 \mathrm{~cm}$ path were prepared for measurement. CB7 $(1 \mathrm{mM})$ was added to the BMVMC $(5 \mu \mathrm{M})$ aqueous solution.

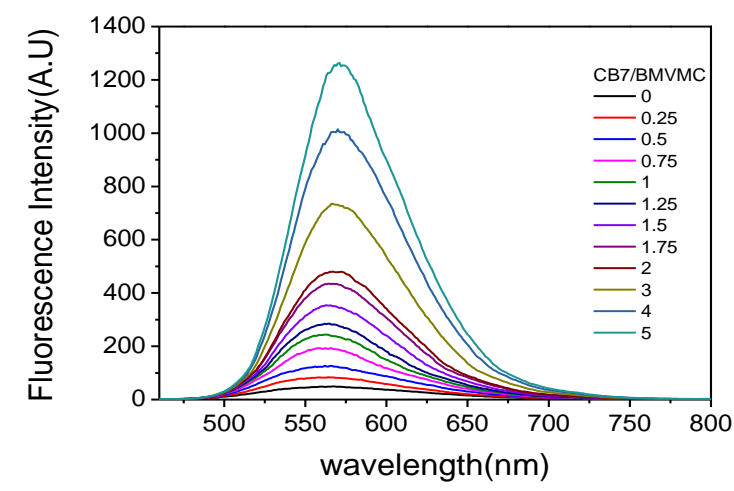

Figure S4. Fluorescence titration spectrum of BMVMC (5 $\mu \mathrm{M})$ titrate CB7 $(1 \mathrm{mM})$. 
5. The quadratic dependence during TPA cross section measurement

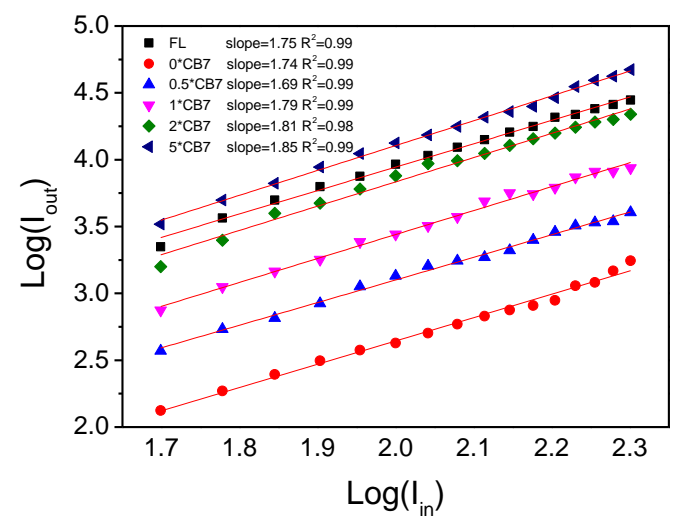

Figure S5. The plot of quadratic dependence for BMVMC in different $\mathrm{CB} 7$ concentration. 
6. The electron paramagnetic resonance (EPR) characterization of CB7/BMVMC aqueous solution

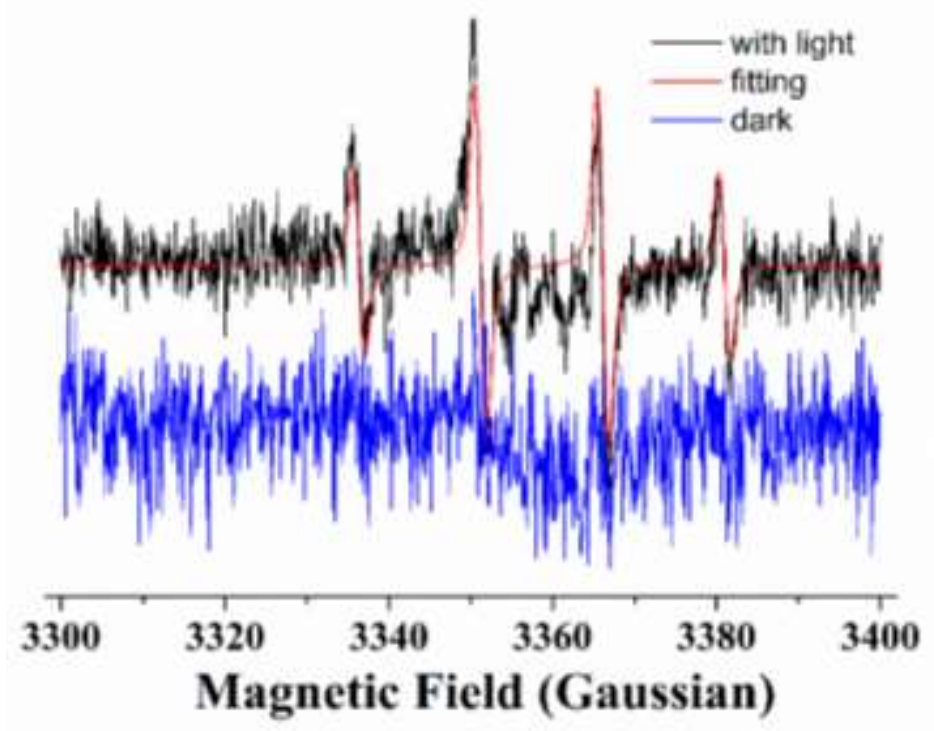

Figure S6. The EPR characterization of CB7/BMVMC aqueous solution at a concentration of 1.5 $\mathrm{mM}$ with 5,5-dimethyl-1-pyrroline-N-oxide (DMPO) as the spin-trapping agent, with the irradiation of the mercury lamp. Fine fractionation of nitrogen and hydrogen are $A_{N} 14.9737 \mathrm{G}$ and $A_{H} 16.6984 \mathrm{G}$, respectively. Dark control means the sample without light irradiation. 
7. Cell viability of CB7/BMVMC aqueous solution
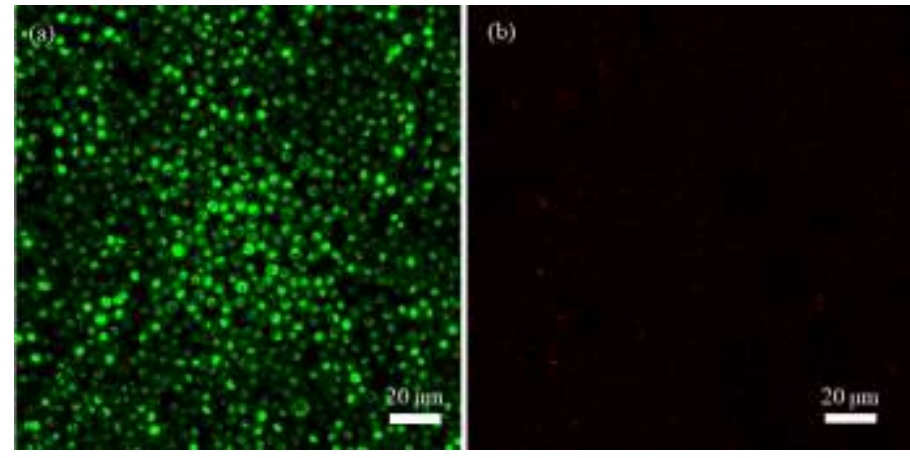

Figure S7. Confocal fluorescence images of the L929 cells co-incubated with Cucurbit[7]uril and BMVMC (a) excited by $488 \mathrm{~nm}$ and (b) incubated after PI $\left(\lambda_{\mathrm{ex}}=561 \mathrm{~nm}\right)$.

We have co-incubated L929 cells with $1 \mathrm{~mL}$ DMEM contained $0.12 \mathrm{wt} \%$ Cucurbit[7] uril (CB7) and BMVMC (equal to the content in cell scaffold) for $24 \mathrm{~h}$. The dead cells are stained by Propidium Iodide (PI, Invitrogen, $\lambda_{\mathrm{ex}}=561 \mathrm{~nm}, 13 \mu \mathrm{L}$ of 1.5 $\mathrm{mM}$ in $1 \mathrm{~mL}$ DMEM with L929), and then observed by confocal fluorescence microscopy. BMVMC can stain the nucleus and we can observe the fluorescence images excited by $488 \mathrm{~nm}$ (Figure S7a), we find that the cells grow well. Figure S7b is the confocal fluorescence image of the cells excited by $561 \mathrm{~nm}$ after being stained by PI. There is no obvious red fluorescence and indicates no dead cells. Therefore, we believe CB7 and BMVMC are nontoxic to the living cells at the concentration level in two-photon polymerization of cell scaffolds. 